\title{
Clinical and radiological outcomes of modified posterior closing wedge osteotomy for the treatment of posttraumatic thoracolumbar kyphosis
}

\author{
Dae-Jean Jo, MD, ${ }^{1}$ Yong-Sang Kim, MD, ${ }^{2}$ Sung-Min Kim, MD, ${ }^{1}$ Ki-Tack Kim, MD, ${ }^{3}$ and \\ Eun-Min Seo, MD
}

\begin{abstract}
Departments of ${ }^{1}$ Neurosurgery and ${ }^{3}$ Orthopedic Surgery, Spine Center, Kyung Hee University Hospital at Gangdong, Seoul; 2Department of Neurosurgery, Spine Center, Good Medical Hospital, Guri; and ${ }^{4}$ Department of Orthopedic Surgery, Chunchon Sacred Heart Hospital, Hallym University College of Medicine, Chuncheon, Korea
\end{abstract}

\begin{abstract}
OBJECT Most thoracolumbar fractures have a good healing outcome with adequate treatment. However, posttraumatic thoracolumbar kyphosis can occur in a proportion of thoracolumbar fractures after inappropriate treatment, osteoporosis, or osteonecrosis of the vertebral body. There are several surgical options to correct posttraumatic thoracolumbar kyphosis, including anterior, posterior, and combined approaches, which are associated with varying degrees of success. The aim of this study was to assess the use of a modified closing wedge osteotomy for the treatment of posttraumatic thoracolumbar kyphosis and to evaluate the radiographic findings and clinical outcomes of patients treated using this technique.
\end{abstract}

METHODS Thirteen consecutive patients with symptomatic posttraumatic thoracolumbar kyphosis were treated using a modified closing wedge osteotomy. The mean patient age was 62 years. The kyphosis apex ranged from T-10 to L-2. The sagittal alignment, kyphotic angle, neurological function, visual analog scale for back pain, and Oswestry Disability Index were evaluated before surgery and at follow-up.

RESULTS The mean preoperative regional angle was $27.4^{\circ}$, and the mean correction angle was $29.6^{\circ}$. Sagittal alignment improved with a mean correction rate of $58.3 \%$. The mean surgical time was 275 minutes, and the mean intraoperative blood loss was $1585 \mathrm{ml}$. The intraoperative complications included 2 dural tears, 1 nerve root injury, and 1 superficial wound infection. The mean visual analog scale score for back pain improved from 6.6 to 2 , and the Oswestry Disability Index score decreased from 55.4 to 22.6 at the last follow-up. All patients achieved bony anterior fusion based on the presence of trabecular bone bridging at the osteotomy site.

CONCLUSIONS The modified posterior closing wedge osteotomy technique achieves satisfactory kyphosis correction with direct visualization of the circumferentially decompressed spinal cord, as well as good fusion with less blood loss and fewer complications. It is an alternative method for treating patients with posttraumatic thoracolumbar kyphosis.

http://thejns.org/doi/abs/10.3171/2015.1.SPINE131011

KEY WORDS thoracolumbar fracture; posttraumatic thoracolumbar kyphosis; modified posterior closing wedge osteotomy; deformity

$\mathrm{M}$ OST thoracolumbar fractures have a good healing outcome when treated adequately. Unstable thoracolumbar fractures are managed using urgent surgical treatment, whereas less severe thoracolumbar fractures are managed using bracing and serial imaging to evaluate bony healing and alignment. However, posttraumatic thoracolumbar kyphosis can occur in a proportion of thoracolumbar fractures despite adequate treatment or due to inappropriate or delayed treatment. ${ }^{24,29}$

Posttraumatic thoracolumbar kyphosis could be caused by factors related to treatment of the injury, such as delayed treatment, inadequate immobilization, weight bearing too early, incorrect surgical procedure and/or fixation (posterior-only surgery, short fusion segment, or prior

ABBREVIATIONS AIS = American Spinal Injury Association Impairment Scale; AP = anteroposterior; ODI = Oswestry Disability Index; SPO = Smith-Petersen osteotomy; VAS = visual analog scale.

SUBMITTED January 3, 2014. ACCEPTED January 15, 2015.

INCLUDE WHEN CITING Published online July 3, 2015; DOI: 10.3171/2015.1.SPINE131011.

DISCLOSURE The authors report no conflict of interest concerning the materials or methods used in this study or the findings specified in this paper. 
laminectomy), and improper choice of internal fixation devices, and also by factors unrelated to treatment, such as osteoporosis, osteonecrosis of the vertebral body, or Charcot spine. $15,20,23,29$

Pathological changes in posttraumatic thoracolumbar kyphosis include muscle or disc degeneration, canal compromise, and local instability. Patients with posttraumatic thoracolumbar kyphosis present with a variety symptoms, including local muscle fatigue or pain, focal deformity, and neurological deficits. ${ }^{7,9,13,29,31}$ Therefore, pain intractable to conservative treatment, progressive deformity, or progressive neurological deficits are the main indications for surgical intervention.

There are several surgical procedures that can be used to correct kyphosis. ${ }^{10,17,26,27}$ Since the Smith-Petersen osteotomy (SPO) technique was first described by SmithPetersen in 1945, anterior, posterior, or combined anterior and posterior procedures for correcting thoracolumbar kyphosis have been advocated by different authors, with varying degrees. ${ }^{5,18,23,25}$ However, the ideal management remains controversial.

The aim of this retrospective study was to illustrate the technique of modified closing wedge osteotomy for the treatment of posttraumatic thoracolumbar kyphosis and to evaluate the radiological findings and clinical outcomes of patients treated using this technique.

\section{Methods \\ Patients}

Thirteen consecutive patients with symptomatic posttraumatic thoracolumbar kyphosis were treated using modified closing wedge osteotomy at our hospital between May 2010 and May 2012. This study was performed with the approval of the ethics committee of our hospital. The patients included 3 males and 10 females, with a mean age at the time of operation of 62 years (range 44-75 years). The mean follow-up period was $22 \pm 17$ months (range 12-32 months).

The mean delay between the initial fracture and kyphosis correction was $29 \pm 17$ months (range 8-63 months). All cases had been managed conservatively (no previous surgical treatment), and the injured vertebra was the kyphotic apex vertebra as well as the osteotomy level (T-10 in 1 case, T-11 in 1 case, T-12 in 3 cases, L-1 in 6 cases, and $\mathrm{L}-2$ in 2 cases) (Table 1 ).

The main complaint of all patients was back pain, as well as pain, numbness, or weakness of the lower extremities. For patients with neurological deficits, the American Spinal Injury Association Impairment Scale (AIS) grade was $\mathrm{C}$ in 2, D in 5, and $\mathrm{E}$ in 6 patients. All patients received strict and regular conservative treatment for at least 3 months before surgery and had definite surgical indications (pain intractable to conservative treatment, progressive deformity, or progressive neurological deficits).

\section{Operative Technique}

All procedures were performed using somatosensory evoked potential monitoring. Following endotracheal intubation, patients were placed prone on a radiolucent operating table. Transverse chest and hip/thigh pads were used to maintain maximum extension of the lumbar spine to facilitate intraoperative osteoclasis throughout the osteotomy.

A posterior midline incision was made spanning from 2 or 3 levels above the fracture site to 2 or 3 levels below it, and a subperiosteal dissection was performed from the spinous process to the tip of the transverse process at all predetermined levels.

With C-arm fluoroscopic guidance, monoaxial pedicle screws were then inserted 2 or 3 levels above and 2 or 3 levels below the osteotomy site. The selection of 2 versus 3 levels above and below the instrumentation was based on the bone strength (age and bone mineral density score). Five cases involved 2 levels, and 8 cases involved 3 levels above and below the instrumentation. The modified closing wedge osteotomy was then performed (Fig. 1). Posterior elements of the apical vertebra were removed, and epidural scarring was released carefully if necessary. An extensive posterior decompression of the dura was necessary to prevent invagination of the dura with osteotomy closure. Meticulous subperiosteal dissection was performed down to the lateral wall of the apical vertebra. Care was taken to protect the exiting nerve root and segmental vessels. Electric cauterization and hemostatic gauze were used to control bleeding of any segmental vessels injured during dissection. A transpedicular decancellous procedure, which is similar to the "egg-shell" technique, was then performed within the apical vertebral body using a pedicle probe and drill to create a wedge resection space toward the upper segment of the damaged intervertebral disc. During this process, a probe was used to check the depth of the bone resection under fluoroscopy. When an adequate amount of the vertebral body was removed, the bilateral cortical bone of the apical vertebra was removed using a rongeur to create an operative window, which can provide sufficient space for removing the upper damaged disc. The posterior cortical bone, upper endplate, and intervertebral disc of the apical vertebral body were then removed (Fig. 1). Temporary rods were used to prevent any uncontrolled closure of the osteotomy space, and the size of wedge space was adjusted according to the surgical plan.

Finally, the wedge osteotomy space was closed gradually and smoothly while confirming no residual soft or bony compression at the resected margin. No further anterior support was needed. Adequate auto- and allograft bone was then placed in the intertransverse processes in each patient. Suction drains were placed, and the wound was closed in layers.

Postoperatively, the patients were allowed out of bed using a customized thoracolumbar orthosis 24 hours after surgery. The orthosis was used for 3 months.

\section{Radiological Evaluation and Clinical Assessments}

Radiological evaluations were based on standing anteroposterior (AP) and lateral views, flexion and extension lateral views, and 3D CT scans. Magnetic resonance imaging was performed to analyze the influence of surgery on the spinal canal. Kyphotic deformity was assessed on lateral radiographs using the Cobb method. Regional angles between the superior endplate of the vertebra above the apical (wedged) vertebra and the inferior endplate of 
TABLE 1. Demographic data

\begin{tabular}{cclccc}
\hline $\begin{array}{c}\text { Case } \\
\text { No. }\end{array}$ & Sex/Age $(\mathrm{yrs})$ & \multicolumn{1}{c}{ Initial Injury } & $\begin{array}{c}\text { Kyphotic Apex/ } \\
\text { Osteotomy Level }\end{array}$ & $\begin{array}{c}\text { Delay Btwn Initial Injury \& Kyphosis } \\
\text { Correction (mos) }\end{array}$ & Follow-Up (mos) \\
\hline 1 & F/66 & T-12 compression fracture & T-12 & 8 & 32 \\
\hline 2 & F/75 & L-1 compression fracture & L-1 & 18 & 30 \\
\hline 3 & F/54 & L-2 burst fracture & L-2 & 11 & 27 \\
\hline 4 & M/75 & L-2 burst fracture & L-2 & 26 & 26 \\
\hline 5 & F/64 & T-10 compression fracture & T-10 & 22 & 24 \\
\hline 6 & F/66 & L-1 compression fracture & L-1 & 63 & 24 \\
\hline 7 & F/60 & T-12 compression fracture & T-12 & 40 & 22 \\
\hline 8 & F/54 & T-11 burst fracture & T-11 & 28 & 22 \\
\hline 9 & M/64 & L-1 compression fracture & L-1 & 38 & 20 \\
\hline 10 & F/70 & L-1 compression fracture & L-1 & 32 & 18 \\
\hline 11 & F/44 & T-12 burst fracture & T-12 & 58 & 15 \\
\hline 12 & M/45 & L-1 burst fracture & L-1 & 19 & 13 \\
\hline 13 & F/70 & L-1 compression fracture & L-1 & 15 & 12 \\
\hline
\end{tabular}

the apical vertebra below were measured preoperatively, immediately after surgery, and at the final follow-up. The sagittal spine alignment was evaluated as the distance from the C-7 plumb line to the posterosuperior corner of S-1 or the sacral promontory.

A negative sagittal imbalance occurred when the C-7 plumb line was posterior to the posterior-superior corner of $\mathrm{S}-1$, and a positive sagittal balance occurred when the C-7 plumb line was anterior to the sacral promontory. If the C-7 plumb line fell between the posterior-superior

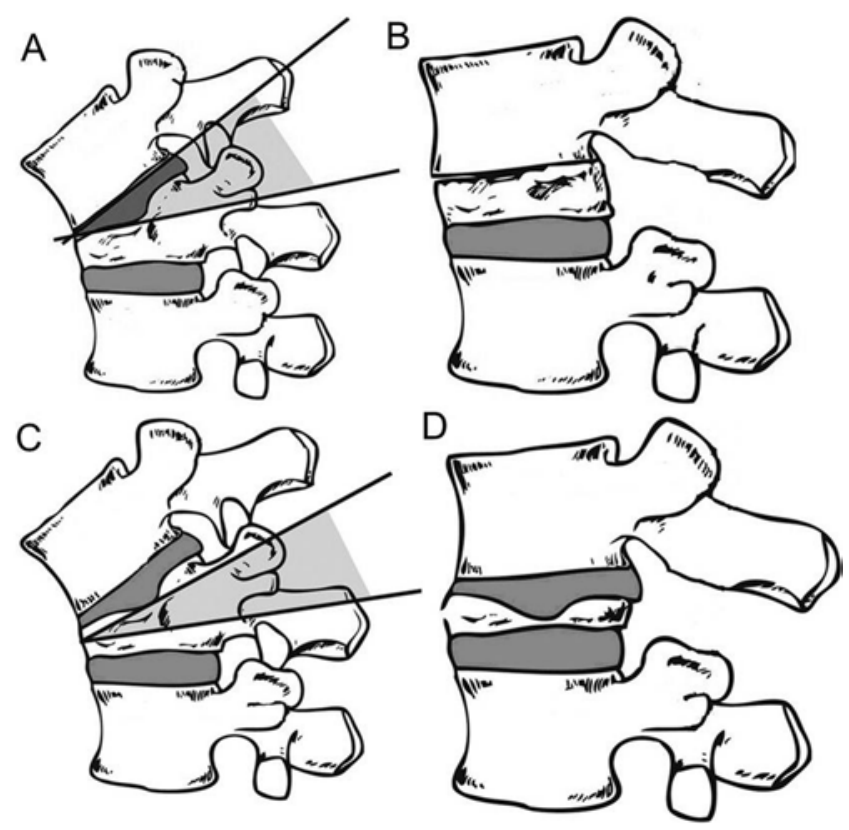

FIG. 1. Modified posterior closing wedge osteotomy. A: The injured intervertebral disc is removed and a portion of the compressed wedge vertebra is left. B: Anterior spinal column achieves bone-on-bone solid fusion. Transpedicular subtraction osteotomy. C: More bone is removed than in modified posterior closing wedge osteotomy. D: The injured intervertebral disc may still descend into the cancellous bone, which can result in nonsolid anterior fusion. Copyright Eun-Min Seo. Published with permission. corner of S-1 and the sacral promontory, a well-balanced condition was indicated. Radiological assessment of fusion at follow-up was performed based on the presence of trabecular bone bridging at the site of osteotomy.

Surgical time, operative blood loss, functional improvement, and complications, including intraoperative and early postoperative events, were assessed using chart reviews. Clinical outcome was measured using the Oswestry Disability Index (ODI), and back pain was assessed using a visual analog scale (VAS) preoperatively and at final follow-up. ${ }^{8}$ The AIS for neurological deficits was used before surgery and at follow-up.

\section{Statistical Analysis}

Measurements were performed by 2 independent observers using the PACS system ( $\pi$ view, Infinitt). The intraobserver and interobserver agreement rate and $\kappa$ values were calculated to assess errors between the 2 observers. For statistical analysis, SPSS (version 17.0, IBM) was used, and a $\mathrm{p}$ value $<0.05$ was considered significant.

\section{Results}

The interobserver agreement rate was $87 \%$ (mean $\kappa=$ 0.65 ), and intraobserver agreement rate was $93 \%$ (mean $\kappa$ $=0.77$ ). The intraobserver and interobserver error analyses showed good agreement.

The mean preoperative regional angle was $27.4^{\circ} \pm 5.8^{\circ}$ $\left([ \pm \mathrm{SD}]\right.$ range $\left.19^{\circ}-42^{\circ}\right)$. Immediately after the operation, the mean regional angle was $-2.2^{\circ} \pm 5.1^{\circ}$ (range $-10^{\circ}$ to $4^{\circ}$ ), and it was $-1.3^{\circ} \pm 4.9^{\circ}$ (range $-9^{\circ}$ to $4^{\circ}$ ) at the last follow-up. The mean correction angle was $29.6^{\circ} \pm 5.3^{\circ}$ (range $20^{\circ}-41^{\circ}$ ). The difference in the mean preoperative and postoperative regional angles was statistically significant $(\mathrm{p}=0.021)$. No obvious correction loss was noted at the last follow-up. The sagittal vertical axis was decreased from a preoperative mean of $2.4 \pm 2.75 \mathrm{~cm}$ (range -1.0 to $10 \mathrm{~cm}$ ) to the last follow-up mean of $1.0 \pm 1.12 \mathrm{~cm}$ (range -1.1 to $2.6 \mathrm{~cm}$ ) with an average correction rate of $58.3 \%$ (Table 2). 
TABLE 2. Radiological outcome in the 13 patients

\begin{tabular}{crrrrrr}
\hline & \multicolumn{3}{c}{ Regional Kyphosis Angle $\left(^{\circ}\right)$} & & \multicolumn{3}{c}{ Sagittal Vertical Axis (cm) } \\
\cline { 2 - 3 } \cline { 6 - 7 } Case No. & Preop & Postop & Last FU & & Preop & Last FU \\
\hline 1 & 30.5 & -2.5 & -0.1 & & 2.46 & 1.59 \\
\hline 2 & 27.6 & -7.1 & -6.6 & & 4.62 & 2.64 \\
\hline 3 & 19.1 & -10.1 & -8.8 & & -0.95 & -0.95 \\
\hline 4 & 22.4 & -8.4 & -7.1 & & 0.53 & -1.06 \\
\hline 5 & 25.5 & -0.5 & 1.0 & & 1.4 & 0.8 \\
\hline 6 & 31.2 & 2.0 & 2.5 & & 0.7 & 0.7 \\
\hline 7 & 28.3 & 2.8 & 3.0 & & 2.8 & 1.5 \\
\hline 8 & 27.8 & 3.8 & 4.2 & & 1.1 & 0.5 \\
\hline 9 & 28.8 & -3.5 & -2.2 & & 2.1 & 1.1 \\
\hline 10 & 23.3 & 3.7 & 4.0 & & 4.4 & 2.3 \\
\hline 11 & 29.6 & 0.5 & 0.9 & & 1.3 & 0.6 \\
\hline 12 & 20.5 & -9.4 & -8.8 & 0.9 & 0.9 \\
\hline 13 & 41.6 & 0.5 & 1.2 & 10 & 2.3 \\
\hline
\end{tabular}

$\mathrm{FU}=$ follow up.

The mean surgical time was 275 minutes (range 185360 minutes), with a mean intraoperative blood loss of $1585 \mathrm{ml}$ (range 800-3000 ml) (Table 3). Some patients exhibited a large amount of intraoperative blood loss due to segmental vessel injury. Meticulous subperiosteal dissection was performed to expose the lateral wall of the apical vertebra. However, the segmental vessels were often injured during dissection because of adhesion of inflammatory granulation tissue around the apical vertebra. Consequently, care was taken to protect the segmental vessels. Intraoperative complications included 2 dural tears, 1 nerve root injury, and 1 superficial wound infection. All dural tears were repaired primarily and did not result in adverse sequelae. The nerve root injury resulted in postsurgical radiculitis and was managed conservatively. The superficial wound infection was treated successfully using debridement, primary closure over drains, and antibiotic therapy. There were no other severe complications.

The mean VAS back pain score decreased from a mean of $6.6 \pm 1.0$ (range 5-8) preoperatively to $2 \pm 0.7$ (range $1-3)$ at the last follow-up ( $p=0.001)$, and the ODI score decreased from a mean value of $55.4 \pm 16.0$ (range 35-87) preoperatively to $22.6 \pm 5.8$ (range 16-35) at the last follow-up ( $\mathrm{p}=0.001)($ Table 3$)$.

Some patients exhibited improved neurological function after surgery. The AIS grade was D in 6 patients and Grade E in 7 patients at the last follow-up. The preoperative AIS grade was $\mathrm{C}$ in 2 patients and improved to Grade D. The preoperative AIS grade was D in 5 patients; 2 of these patients improved to Grade E. However, 1 patient with preoperative Grade E worsened in neurological function due to intraoperative nerve root injury, to Grade D (Table 3).

All patients achieved bony fusion, which was confirmed based on the presence of trabecular bone bridging at the osteotomy and intertransverse processes site using radiographic evaluations, including AP and lateral views, and CT scans (Figs. 2 and 3).

\section{Discussion}

Most thoracolumbar fractures have a good healing outcome after adequate treatment. However, posttraumatic thoracolumbar kyphosis can develop in a proportion of thoracolumbar fractures despite adequate treatment. In addition, posttraumatic thoracolumbar kyphosis can develop due to delayed or inappropriate treatment methods..$^{24,29}$

Posttraumatic thoracolumbar kyphosis might be caused by factors related to treatment of the injury, such as delayed treatment, inadequate immobilization, weight bearing too early, incorrect surgical procedure and fixation (posterioronly surgery, short fusion segment, or prior laminectomy), or improper choice of internal fixation devices, as well as by factors unrelated to treatment, such as osteoporosis, osteonecrosis of the vertebral body, or Charcot spine., ${ }^{4,24,29}$

In our study, posttraumatic thoracolumbar kyphosis was caused by inappropriate conservative treatment, osteoporosis, or osteonecrosis of the vertebral body in 13 patients. Posttraumatic thoracolumbar kyphosis can lead to the gradual collapse of the injured vertebra because of

TABLE 3. Clinical outcome data in the 13 patients

\begin{tabular}{|c|c|c|c|c|c|c|c|c|}
\hline \multirow[b]{2}{*}{ Case No. } & \multirow[b]{2}{*}{ Blood Loss (ml) } & \multirow[b]{2}{*}{ Op Time (mins) } & \multicolumn{3}{|c|}{ Preop } & \multicolumn{3}{|c|}{ Last FU } \\
\hline & & & AIS Score & Back Pain VAS Score & ODI & AIS & Back Pain VAS Score & ODI \\
\hline 1 & 2500 & 315 & $\mathrm{E}$ & 7 & 63 & E & 2 & 25 \\
\hline 2 & 1600 & 295 & D & 8 & 72 & D & 3 & 31 \\
\hline 3 & 3000 & 360 & $E$ & 7 & 51 & E & 2 & 19 \\
\hline 4 & 1500 & 305 & C & 5 & 44 & D & 1 & 17 \\
\hline 5 & 1200 & 185 & $E$ & 6 & 39 & E & 2 & 16 \\
\hline 6 & 1000 & 240 & D & 7 & 51 & D & 2 & 23 \\
\hline 7 & 800 & 245 & D & 6 & 45 & $\mathrm{E}$ & 2 & 24 \\
\hline 8 & 900 & 270 & $E$ & 6 & 54 & E & 2 & 20 \\
\hline 9 & 1500 & 185 & $\mathrm{D}$ & 8 & 74 & D & 3 & 27 \\
\hline 10 & 1800 & 315 & C & 5 & 67 & D & 1 & 22 \\
\hline 11 & 1200 & 275 & $E$ & 7 & 38 & $E$ & 2 & 19 \\
\hline 12 & 800 & 270 & $\mathrm{D}$ & 6 & 35 & $E$ & 1 & 16 \\
\hline 13 & 2800 & 320 & $E$ & 8 & 87 & D & 3 & 35 \\
\hline
\end{tabular}



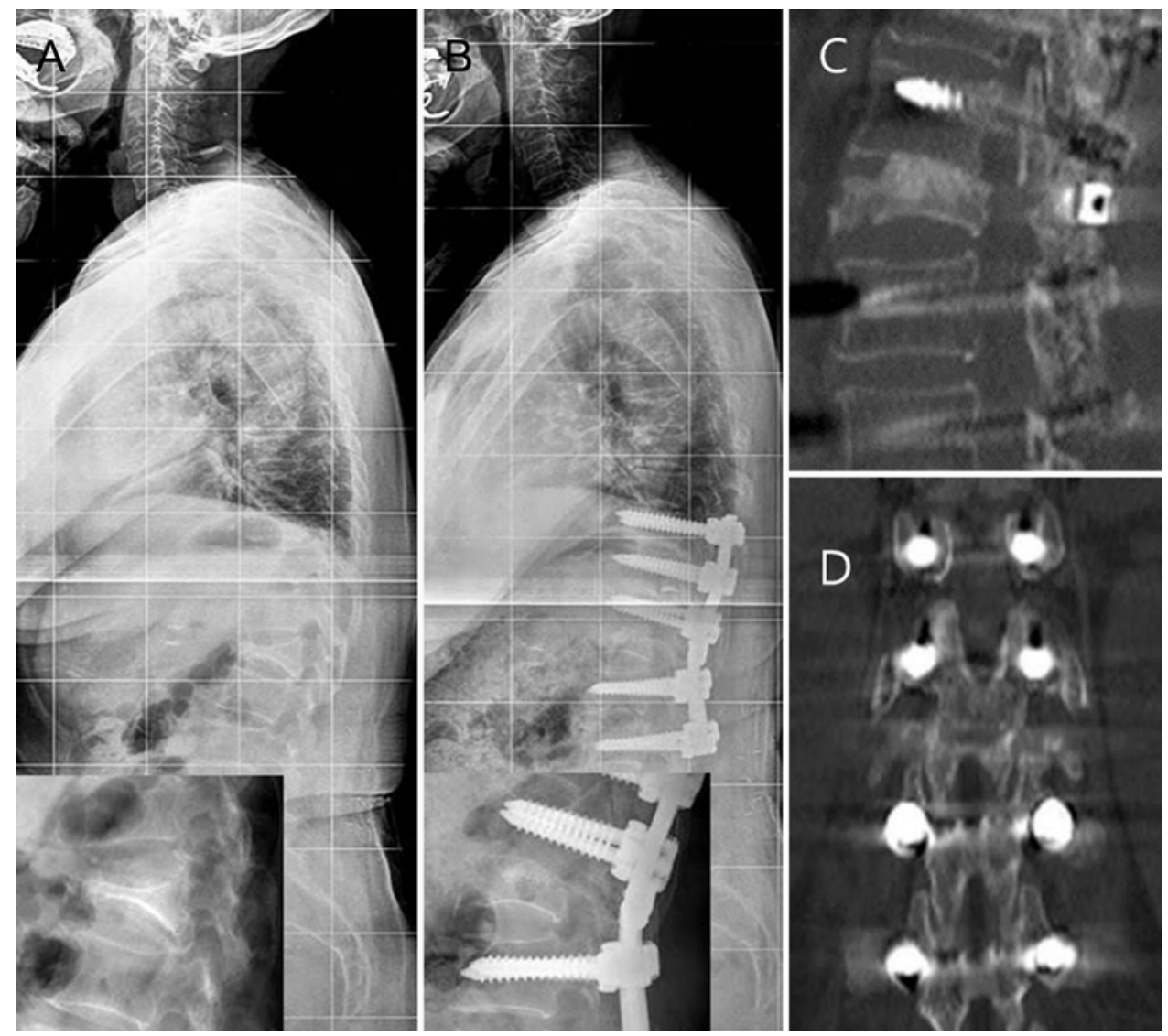

FIG. 2. A: Lateral standing radiographs obtained in a 75-year-old woman who had severe back pain and both leg weakness shows L-1 compression fracture and posttraumatic thoracolumbar kyphosis (regional angle $27.6^{\circ}$ ). She was treated using modified posterior closing wedge osteotomy. B: Postoperative lateral standing radiographs show good sagittal alignment (regional angle $\left.-6.6^{\circ}\right)$. C and D: At follow-up 1.5 years after surgery, CT scans show bony fusion at the osteotomy site.

the direct force on the anterior and middle column of the spine.

The biomechanical factors involved in the progression of the posttraumatic thoracolumbar kyphosis include an increase in the lever arm at the center of the gravity line with respect to the wedged vertebra..$^{30}$ The anterior elements of the spine fail under compression, and the posterior elements fail under tension. This results in an increase in the eccentric loads and increased wedging and kyphosis. With increased kyphosis, the intractable pain might derive from the site of the deformity itself, the injured disc, a bony nonunion, or the lordotic compensation above and below the deformity site where additional stresses are placed on the respective facet joints. In addition, the direct compression of the spinal cord caused by the apical vertebral body, degenerative discs, hypertrophic ligaments, and kyphosis stretching the spinal cord might result in neurological symptoms. . $^{14,23,32}$

The surgical treatment of posttraumatic thoracolumbar kyphosis remains controversial. Whether the angle of kyphosis is the key factor indicating surgical therapy is undecided. Bedbrook reported that there is no association between kyphosis and pain, and patients reported no pain even when the angle of kyphosis was greater than $40^{\circ} .1$ However, Gertzbein and Harris considered that when the angle of kyphosis exceeded $30^{\circ}$, the risk of pain increased significantly and surgical correction was necessary. ${ }^{9}$ Chen et al. recommended that any kyphosis case with a kyphotic angle of more than $20^{\circ}$ requires surgical correction. ${ }^{6}$ In our study, the mean angle of kyphosis was more than $27.4^{\circ}$, and the patients exhibited abnormal posture, localized symptoms, and/or neurological deficits. Another controversial issue is whether patients with nerve damage and longer fracture histories without local symptoms should be treated using surgery. Bohlman et al. reported that for patients with a fracture history of less than 2 years, surgical therapy obtains good therapeutic results. ${ }^{2}$

Zhang et al. found that among patients with even longer histories of fracture, 58\% exhibited improved neurological function after correction and decompression..$^{33}$ The duration of the fracture is not an absolute factor that affects the therapeutic outcome of surgical management. Therefore, surgical treatment should be considered in cases of pain that is intractable to nonsurgical management, progressive deformity, or deteriorating neurological status.

The purpose of surgery is to restore the spinal sequence, relieve the direct compression of the spinal cord, reconstruct spinal balance and stability, improve symptoms, and prevent deformity. Surgery to correct posttraumatic thoracolumbar kyphosis has taken several forms, including anterior, posterior, and combined approaches..$^{10,17,26,27}$ Posterior spinal fusion alone in the presence 

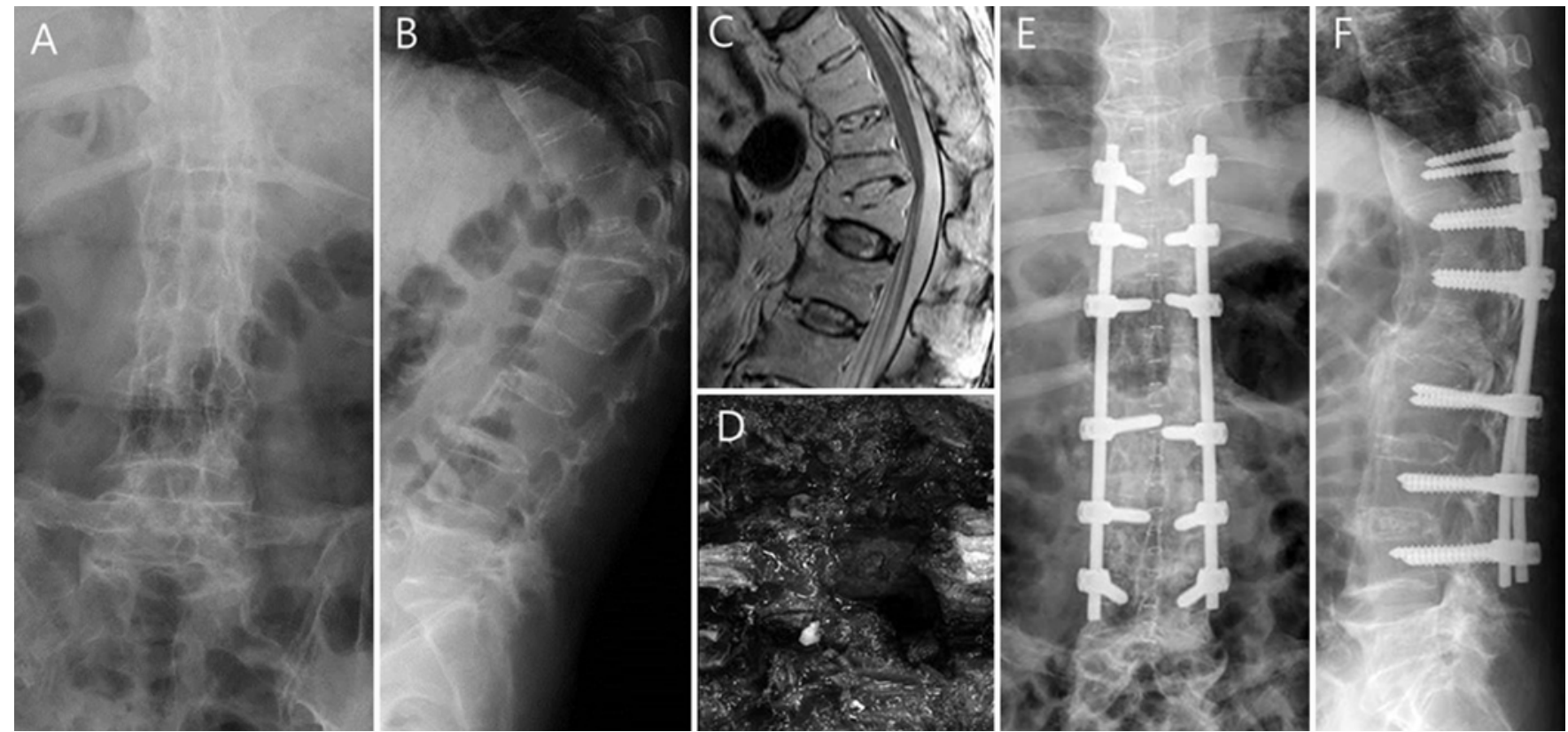

FIG. 3. A and B: AP and lateral standing radiographs obtained in a 70 -year-old woman showing L-1 compression fracture and posttraumatic thoracolumbar kyphosis (regional angle $41.6^{\circ}$ ). C: MR image shows severe spinal cord compression. D: The patient was treated using modified posterior closing wedge osteotomy. E and F: Fifteen months later, postoperative AP and lateral standing radiographs show good sagittal alignment (regional angle $1.2^{\circ}$ ) and bony fusion at the osteotomy site.

of existing kyphosis is not considered sufficient to treat posttraumatic kyphosis, and some studies have suggested that the anterior approach is better for vertebral compression fractures because the pathology is anterior. ${ }^{12}$ Kaneda et al. and Kostuik and Matsusaki have reported successful results for anterior decompression and stabilization using anterior instrumentation for posttraumatic thoracolumbar kyphosis. ${ }^{12,16}$ However, others have suggested that anterior decompression and fusion should not be used alone to correct rigid kyphosis and have recommended additional posterior instrumentation and fusion to increase the rate of arthrodesis. ${ }^{19}$ Therefore, there is a consensus that both anterior and posterior factors should be managed to sufficiently restore the alignment and obtain fusion in posttraumatic thoracolumbar kyphosis. ${ }^{23,28}$ Böhm et al. combined dorsal decompression and fixation with ventral osteotomy and grafting and achieved a mean angular correction of $22.5^{\circ}$ in 40 patients. ${ }^{3}$ However, a 2 -stage or 3 -stage supineprone-supine procedure is associated with more incisions, complications, and blood loss.

Currently, a single posterior approach is used most often for correction of the posttraumatic kyphosis. Posterior approach surgical techniques include SPO, posterior closing wedge osteotomy or pedicle subtraction osteotomy, and single-stage posterior vertebral column resection..$^{10,17,26,27}$ Smith-Petersen osteotomy is used mainly for posterior column removal, shortening the posterior column, and lengthening the anterior column but it is associated with more neurovascular complications, lower fusion rate, and increased incidence of loss of correction than posterior subtraction osteotomy or posterior closing wedge osteotomy. Posterior closing wedge osteotomy or pedicle subtraction osteotomy shortens the posterior column but does not lengthen the anterior column, lowering the risk of spinal cord injury and achieving high fusion rates and great correction angles.

Posterior closing wedge osteotomy or pedicle subtraction osteotomy in a single posterior approach was advocated by Heinig. ${ }^{11}$ One of the great advantages of the circumspinal decompression is to allow a clear visibility of the spinal cord and to ensure the absence of dangerously positioned structures around the spinal cord. Heinig used a modification of the Michele and Krueger approach and proposed the eggshell procedure to correct kyphosis, which consists of a closing wedge osteotomy. ${ }^{11,21}$ The principle is to weaken the vertebra anterior to the cord, remove all parts of the posterior elements, and produce a controlled compression fracture, while carefully observing the spinal cord and roots. This approach is thought to be reasonable because not only is deformity corrected but also the moment arm of kyphotic deformity is shortened, without stretching the spinal cord. The major vessels around the spine are also safe. This procedure was later modified by Gertzbein and Harris to correct posttraumatic kyphosis, and could achieved a mean of $30^{\circ}$ sagittal correction. ${ }^{9}$ Kawahara et al. described a technique of circumspinal decompression and closing-opening osteotomy using a single posterior approach to correct an angular kyphotic deformity. ${ }^{13}$ Anterior decompression of the spinal cord is possible by costotransversectomy, and patients with or without neurological deficits are considered as suitable candidates for the procedure. In the 7 patients treated, localized kyphosis was reduced from a mean of $67^{\circ}$ to $18^{\circ}$, and the sagittal alignment was improved. Suk et al. compared the surgical results between combined anterior-posterior procedures and posterior closing wedge osteotomy in posttraumatic kyphosis patients and believed that posterior closing wedge osteotomy may result in bet- 
ter correction, shorter operative time, and less blood loss compared with anterior-posterior surgery. ${ }^{28}$ Therefore, the posterior closing wedge osteotomy procedure has been widely performed in posttraumatic kyphosis, with excellent results without neurological deterioration.

However, a posterior closing wedge osteotomy procedure is not always reasonable in cases of the posttraumatic thoracolumbar kyphosis with a severely wedged vertebra. The thoracolumbar junction is a transition between thoracic kyphosis and lumbar lordosis. The normal sagittal alignment of the thoracolumbar junction is straight, and there is no additional support from the rib cage. As such, there is a tendency for the injured vertebrae to collapse gradually and wedge in posttraumatic thoracolumbar kyphosis. Zhang et al. described that the pedicle region, and the posterior part of the upper endplate and disc of the injured vertebra are usually the apex of the posttraumatic thoracolumbar kyphosis, which compresses on the spinal cord. ${ }^{34}$ When a regular posterior closing wedge osteotomy or pedicle subtraction osteotomy is attempted at the site of the severely wedged vertebra, it is inevitable that the collapsed upper endplate is removed, and the lower surface of the injured upper disc is exposed. When the closing procedure is attempted, the damaged upper disc will rest on the cancellous bone of the remaining vertebra. The damaged upper disc might induce back pain. Furthermore, bony fusion is difficult to achieve in this situation. Therefore, the modified posterior closing wedge osteotomy technique (posterior closing wedge osteotomy with removing the injured upper endplate and upper disc) can achieve effective correction of kyphosis and solid fusion between the inferior bony endplate of the upper vertebra and the rectangular lower half of the injured vertebra, which is of great importance for back pain relief.

A correction angle within $45^{\circ}$ is considered to be safe. Heary and Bono used pedicle subtraction osteotomy to obtain a mean correction angle of $51^{\circ}$ without postoperative complications..$^{10} \mathrm{Wu}$ et al. also reported a single-staged posterior approach, posterior decancellation osteotomy, and achieved a mean correction of $38.8^{\circ}$ in rigid posttraumatic kyphosis patients. ${ }^{31}$ Lehmer et al. reported on 38 patients who underwent pedicle subtraction osteotomy surgery, with a $93 \%$ correction rate, and a $100 \%$ fusion rate at final follow-up. ${ }^{18}$ Qiu et al. used pedicle subtraction osteotomy in 17 cases of kyphosis deformity caused by old thoracolumbar vertebral fractures and obtained a mean correction angle of $40^{\circ}$ and a $100 \%$ fusion rate with a marked improvement in clinical symptoms and neurological function..$^{22}$

In our study, 13 patients were treated using a modified posterior closing wedge osteotomy; the maximum correction angle was $41^{\circ}$ and the mean correction angle was $29.6^{\circ}$. Clinical symptoms were improved significantly by the time of the final follow-up. Neurological function improved from AIS Grade C to D in 2 patients and from AIS Grade $\mathrm{D}$ to $\mathrm{E}$ in 2 patients; however, 1 patient with a preoperative AIS Grade E had worsening neurological function to AIS Grade D due to intraoperative nerve root injury.

Our study had limitations, including a small sample size (13 patients) and a short follow-up period in some of the patients.

\section{Conclusions}

The surgical treatment of posttraumatic thoracolumbar kyphosis is a challenge. Although the modified posterior closing wedge osteotomy procedure is technically demanding, it achieves satisfactory kyphosis correction with a direct visualization of the circumferentially decompressed spinal cord, and good fusion with less blood loss and complications. Therefore, the modified posterior closing wedge osteotomy technique is an alternative method in patients with posttraumatic thoracolumbar kyphosis.

\section{References}

1. Bedbrook GM: Treatment of thoracolumbar dislocation and fractures with paraplegia. Clin Orthop Relat Res (112): 2743, 1975

2. Bohlman HH, Kirkpatrick JS, Delamarter RB, Leventhal M: Anterior decompression for late pain and paralysis after fractures of the thoracolumbar spine. Clin Orthop Relat Res (300):24-29, 1994

3. Böhm H, Harms J, Donk R, Zielke K: Correction and stabilization of angular kyphosis. Clin Orthop Relat Res (258):56-61, 1990

4. Buchowski JM, Kuhns CA, Bridwell KH, Lenke LG: Surgical management of posttraumatic thoracolumbar kyphosis. Spine J 8:666-677, 2008

5. Chang KW: Oligosegmental correction of post-traumatic thoracolumbar angular kyphosis. Spine (Phila Pa 1976) 18:1909-1915, 1993

6. Chen ZQ, Li WS, Guo ZQ, Qi Q, Dang GT: [Surgical correction of post-traumatic kyphosis of thoracolumbar spine.] Zhonghua Wai Ke Za Zhi 43:201-204, 2005 (Chinese)

7. Chen ZW, Ding ZQ, Zhai WL, Lian KJ, Kang LQ, Guo LX, et al: Anterior versus posterior approach in the treatment of chronic thoracolumbar fractures. Orthopedics 35:e219e224, 2012

8. Fairbank JC, Pynsent PB: The Oswestry Disability Index. Spine (Phila Pa 1976) 25:2940-2952, 2000

9. Gertzbein SD, Harris MB: Wedge osteotomy for the correction of post-traumatic kyphosis. A new technique and a report of three cases. Spine (Phila Pa 1976) 17:374-379, 1992

10. Heary RF, Bono CM: Pedicle subtraction osteotomy in the treatment of chronic, posttraumatic kyphotic deformity. J Neurosurg Spine 5:1-8, 2006

11. Heinig JA: Eggshell procedure, in Luque ER (ed): Segmental Spinal Instrumentations. Thorofare, NJ: Slack, 1984, pp 221-230

12. Kaneda K, Asano S, Hashimoto T, Satoh S, Fujiya M: The treatment of osteoporotic-posttraumatic vertebral collapse using the Kaneda device and a bioactive ceramic vertebral prosthesis. Spine (Phila Pa 1976) 17 (8 Suppl):S295-S303, 1992

13. Kawahara N, Tomita K, Baba H, Kobayashi T, Fujita T, Murakami H: Closing-opening wedge osteotomy to correct angular kyphotic deformity by a single posterior approach. Spine (Phila Pa 1976) 26:391-402, 2001

14. King AI, Prasad P, Ewing CL: Mechanism of spinal injury due to caudocephalad accleration. Orthop Clin North Am 6:19-31, 1975

15. Knop C, Fabian HF, Bastian L, Blauth M: Late results of thoracolumbar fractures after posterior instrumentation and transpedicular bone grafting. Spine (Phila Pa 1976) 26:8899, 2001

16. Kostuik JP, Matsusaki H: Anterior stabilization, instrumentation, and decompression for post-traumatic kyphosis. Spine (Phila Pa 1976) 14:379-386, 1989

17. Lazennec JY, Neves N, Rousseau MA, Boyer P, Pascal- 
Mousselard H, Saillant G: Wedge osteotomy for treating post-traumatic kyphosis at thoracolumbar and lumbar levels. J Spinal Disord Tech 19:487-494, 2006

18. Lehmer SM, Keppler L, Biscup RS, Enker P, Miller SD, Steffee AD: Posterior transvertebral osteotomy for adult thoracolumbar kyphosis. Spine (Phila Pa 1976) 19:2060-2067, 1994

19. Malcolm BW, Bradford DS, Winter RB, Chou SN: Posttraumatic kyphosis. A review of forty-eight surgically treated patients. J Bone Joint Surg Am 63:891-899, 1981

20. McLain RF, Burkus JK, Benson DR: Segmental instrumentation for thoracic and thoracolumbar fractures: prospective analysis of construct survival and five-year follow-up. Spine J 1:310-323, 2001

21. Michele AA, Krueger FJ: Surgical approach to the vertebral body. J Bone Joint Surg Am 31A:873-878, 1949

22. Qiu Y, Bangping Q, Bin W: Posterior osteotomy for the treatment of thoracic and lumbar fracture delayed postoperative kyphosis curative effect analysis. Chin J Orthop 28:187191,2008

23. Roberson JR, Whitesides TE Jr: Surgical reconstruction of late post-traumatic thoracolumbar kyphosis. Spine (Phila Pa 1976) 10:307-312, 1985

24. Schoenfeld AJ, Wood KB, Fisher CF, Fehlings M, Oner FC, Bouchard K, et al: Posttraumatic kyphosis: current state of diagnosis and treatment: results of a multinational survey of spine trauma surgeons. J Spinal Disord Tech 23:e1-e8, 2010

25. Shufflebarger HL, Clark CE: Thoracolumbar osteotomy for postsurgical sagittal imbalance. Spine (Phila Pa 1976) 17 (8 Suppl):S287-S290, 1992

26. Smith-Petersen M, Larson CB, Aufranc OE: Osteotomy of the spine for correction of flexion deformity in rheumatoid arthritis. J Bone Joint Surg Am 27:1-11, 1945

27. Suk SI, Kim JH, Kim WJ, Lee SM, Chung ER, Nah KH: Posterior vertebral column resection for severe spinal deformities. Spine (Phila Pa 1976) 27:2374-2382, 2002

28. Suk SI, Kim JH, Lee SM, Chung ER, Lee JH: Anterior-pos- terior surgery versus posterior closing wedge osteotomy in posttraumatic kyphosis with neurologic compromised osteoporotic fracture. Spine (Phila Pa 1976) 28:2170-2175, 2003

29. Vaccaro AR, Silber JS: Post-traumatic spinal deformity. Spine (Phila Pa 1976) 26 (24 Suppl):S111-S118, 2001

30. White AA III, Panjabi MM: Practical biomechanics of scoliosis and kyphosis, in Clinical Biomechanics of the Spine, ed 2. Philadelphia: JB Lippincott, 1990, pp 127-168

31. Wu SS, Hwa SY, Lin LC, Pai WM, Chen PQ, Au MK: Management of rigid post-traumatic kyphosis. Spine 21 (Phila Pa 1976):2260-2267, 1996

32. Xi YM, Pan M, Wang ZJ, Zhang GQ, Shan R, Liu YJ, et al: Correction of post-traumatic thoracolumbar kyphosis using pedicle subtraction osteotomy. Eur J Orthop Surg Traumatol 23 (Suppl 1):S59-S66, 2013

33. Zhang F, Tian G, Liu Z: Analyzing the factors on surgery for old thoracolumbar fractures. Chin J Spine Spinal Cord 13:471-473, 2003

34. Zhang X, Zhang X, Zhang Y, Wang Z, Wang Y: Modified posterior closing wedge osteotomy for the treatment of posttraumatic thoracolumbar kyphosis. J Trauma 71:209-216, 2011

\section{Author Contributions}

Conception and design: Seo, Jo. Acquisition of data: all authors. Analysis and interpretation of data: all authors. Drafting the article: Seo. Critically revising the article: Seo, Jo. Reviewed submitted version of manuscript: all authors. Approved the final version of the manuscript on behalf of all authors: Seo. Statistical analysis: Jo, YS Kim. Administrative/technical/material support: Seo. Study supervision: Seo.

\section{Correspondence}

Eun-Min Seo, Department of Orthopedic Surgery, Chuncheon Sacred Heart Hospital, \#153, Kyo-dong, Chuncheon, Kangwondo 200-704, Korea. email: seoem@hallym.or.kr. 